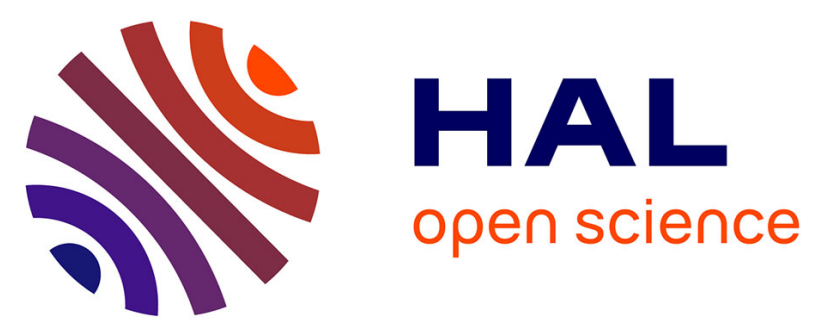

\title{
NOUVEAUX ÉLÉMENTS APPORTÉS A L'ÉTUDE DU SITE MAGDALÉNIEN D'ENVAL (VIC-LE-COMTE, PUY-DE-DOME, FRANCE) ET DU PEUPLEMENT DE LA LIMAGNE D'AUVERGNE
}

Frédéric Surmely, Laure Fontana, Yves Bourdelle, René Llabeuf

\section{To cite this version:}

Frédéric Surmely, Laure Fontana, Yves Bourdelle, René Llabeuf. NOUVEAUX ÉLÉMENTS APPORTÉS A L'ÉTUDE DU SITE MAGDALÉNIEN D'ENVAL (VIC-LE-COMTE, PUY-DE-DOME, FRANCE) ET DU PEUPLEMENT DE LA LIMAGNE D'AUVERGNE. Bulletin de la Société préhistorique française, 1997, 94 (2), pp.172-181. hal-02538717

\section{HAL Id: hal-02538717 https://hal.science/hal-02538717}

Submitted on 9 Apr 2020

HAL is a multi-disciplinary open access archive for the deposit and dissemination of scientific research documents, whether they are published or not. The documents may come from teaching and research institutions in France or abroad, or from public or private research centers.
L'archive ouverte pluridisciplinaire HAL, est destinée au dépôt et à la diffusion de documents scientifiques de niveau recherche, publiés ou non, émanant des établissements d'enseignement et de recherche français ou étrangers, des laboratoires publics ou privés. 


\title{
NOUVEAUX ÉLÉMENTS APPORTÉS A L'ÉTUDE DU SITE MAGDALÉNIEN D'ENVAL (VIC-LE-COMTE, PUY-DE-DOME, FRANCE) ET DU PEUPLEMENT DE LA LIMAGNE D'AUVERGNE
}

\author{
Frédéric SURMELY, Laure FONTANA, Yves BOURDELLE et René LIABEUF
}

\section{RÉSUMÉ}

C'est à l'occasion de travaux d'aménagement effectués dans un abri-sous-roche transformé en cave, à proximité immédiate ou gisement de l'abri Durif, connu notamment pour ses cuures d'art mobilier, qu'a été découvert un petit lambeau d'habitat magdalénien qui a livrè de l'industrie lithique, osseuse, ainsi que des restes fauniques. Ces vestiges, peu nombreux mais particulièrement significatifs, ont fait l'objet d'une étude détaillée forigine de la matière première, stratégies d'approvisionnement, faune) et apportent des renseignements intéressants pour la caractérisation de la présence magdalénienne sur le site d'Enval et dans la Limagne d'Auvergne.

\section{ABSTRACT}

While work was being carried out on a rock shelter transformed into a cave very close to the Abri Durif settlement, known in particular for its mobiliary works of art, a small remnant of Magdalenian occupation was found. It has supplied elements of flint and bone industry as well as remains of fauna. These vestiges, few in number but of particular significance, have been the subject of detailed study lorigins of the raw material, procurement strategies, fauna) and have provided interesting information concerning the characterisation of the Magdalanian presence on the site of Enval and in the Limagne region of Auvergne.

\section{PRÉSENTATION DU SITE D'ENVAL (Y.B.)}

Le site d'Enval est connu pour son occupation magdalénienne, qui semble s'expliquer par une localisation favorable: une longue ligne rocheuse creusée d'abris, orientée plein sud, dominant de quelques mètres un petit ruisseau se jetant dans la vallee de l'Allier, grand axe de circulation (fig. 1). Le site est aujourd'hui occupé par un village, qui a transformé la physionomie originelle de l'escarpement.

Dès 1877, un érudit de la région mentionne la présence d'outils préhistoriques et d'ossements humains dans les abris-sous-roche auxquels sont adossées les maisons (Biélawski, 1887). A l'occasion de travaux dans le bourg, de nouvelles découvertes (outils, foyers, lampe...) sont signalées dans plusieurs cavités voisines (Baudon, 1911 ; Canque, Charvilhat et Goursonnet, 1929).
A partir de 1969, l'un d'entre nous (Y.B.) a fouillé durant près de 20 années dans une des cavités transformées en grange, l'abri Durif, les vestiges d'un gisement qui contenait un abondant mobilier lithique et surtout des objets d'art (plaquettes gravées. Vénus en grès). L'habitat, qui comprenait deux loci distincts recélant chacun plusieurs niveaux d'occupation, est datable de la fin du magdalénien. Le premier locus, le "Sol de la Grange", a livré des dates comprises entre $13510+230 \mathrm{BP}$ (Ly 3675) et $13090+270$ BP (Ly 2046) (Bourdelle, 1987 ; Bourdelle et Daugas, 1969 ; Bourdelle et Merlet, 1991). Les ni-

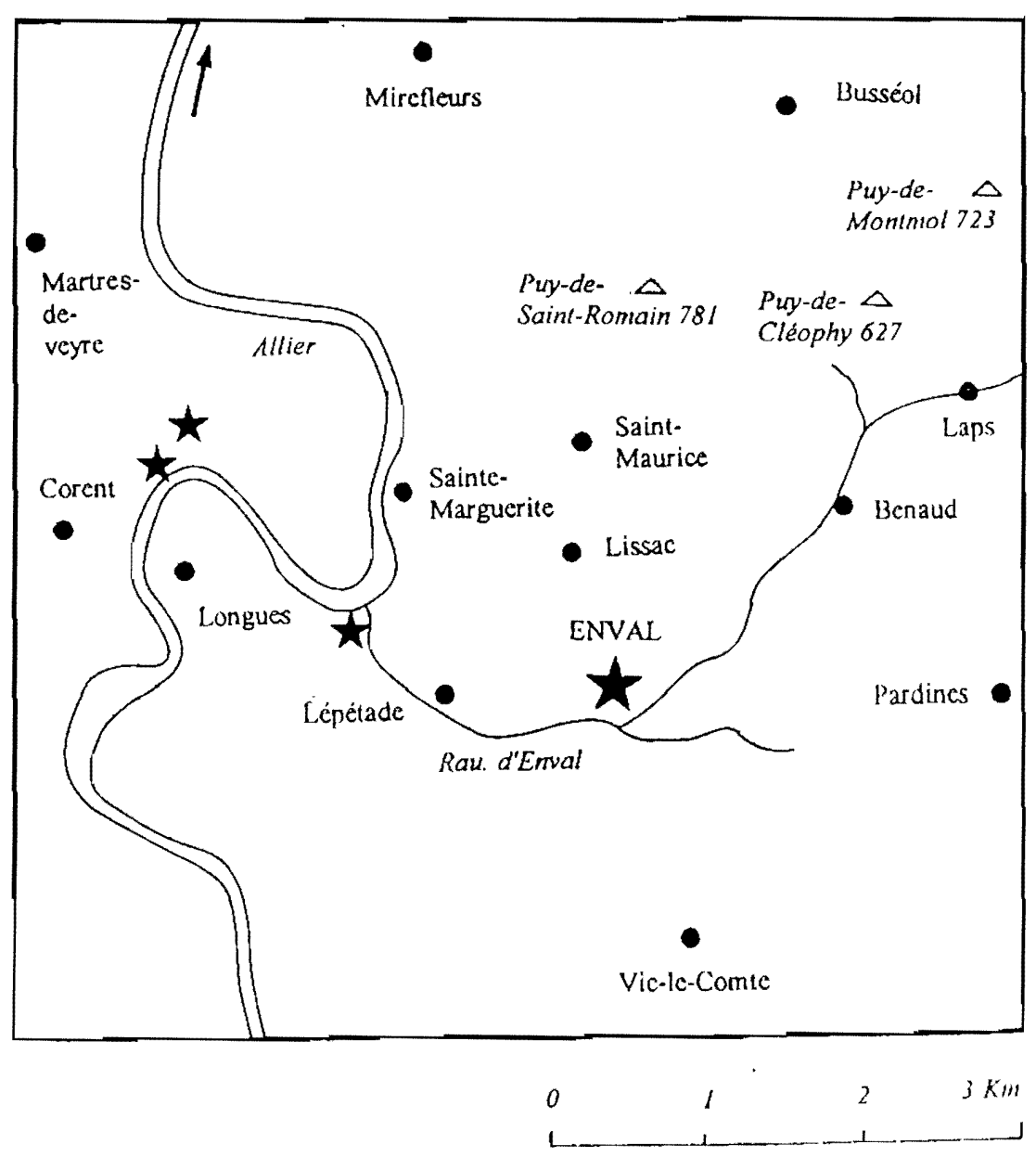

Gisements magdaleniens de plein-air

Fig. 1 - Situation géographique du site d'Enval. 
veaux anciens du locus dit du "Fond de l'Abri" semblent contemporains $(13000+300 \mathrm{BP}-\mathrm{LY} 425)$ et 13700 +380 BP - Ly 727), mais les niveaux supérieurs paraissent plus récents. Le dernier niveau d'habitat est en effet directement scellé par une couche de cendres volcaniques attribuée à la retombée inférieure de Gimeaux, soit $12010+150$ BP Nernet, 1992 ; Raynal et alii, 1994).

Le gisement d'Enval 2, qui rait l'objet de cet article, se trouve à 30 mètres de l'abri Durif, légèrement en contrebas. Comme ce dernier, il s'agit d'un ancien surplomb rocheux transformé en cave. Nous ignorons la nature exacte des relations qui pouvaient unir l'abri d'Enval 2 et l'abri Durif. L'hypothèse d'un unique grand gisement reste envisageable.

Les travaux d'aménagement anciens ont fait disparaître la quasitotalité du remplissage de la cavité d'Enval 2. Seule une petite partie avait été préservée dans le fond et abritait un lambeau de remplissage pléistocène. C'est là que le propriétaire de la maison a découvert de l'outillage en silex au cours de travaux. Alertés par ses soins, nous avons... constaté les dégâts, recueilli le matériel et tamisé les déblais.

Ce contexte explique qu'il ait été impossible de relever la stratigraphie des dépôts. L'existence de plusieurs niveaux est possible. La parfaite homogénéité du mobilier recueilli n'est donc pas assurée.

Toutes les pièces sont recouvertes d'encroûtements rougeâtres ou noirâtres, dus à des eaux d'infiltration riches en oxydes minéraux.

\section{L'INDUSTRIE LITHIQUE (F.S.)}

\section{- Origine de la matière première}

La matière première utilisée à Enval 2 se partage, comme dans tous les gisements du magdalénien de Limagne (Bourdelle, 1987 ; Masson, 1981 ; Daniel, 1979 ; Delporte, 1975), en deux catégories principales (fig. 2).

La première catégorie (rassemblèe sous l'appellation générique de "tertiaire", dans les figures) est constituée par des matières premières locales, issues des calcaires tertiaires de l'Oligocène supérieur et des formations volcano-sédimen-

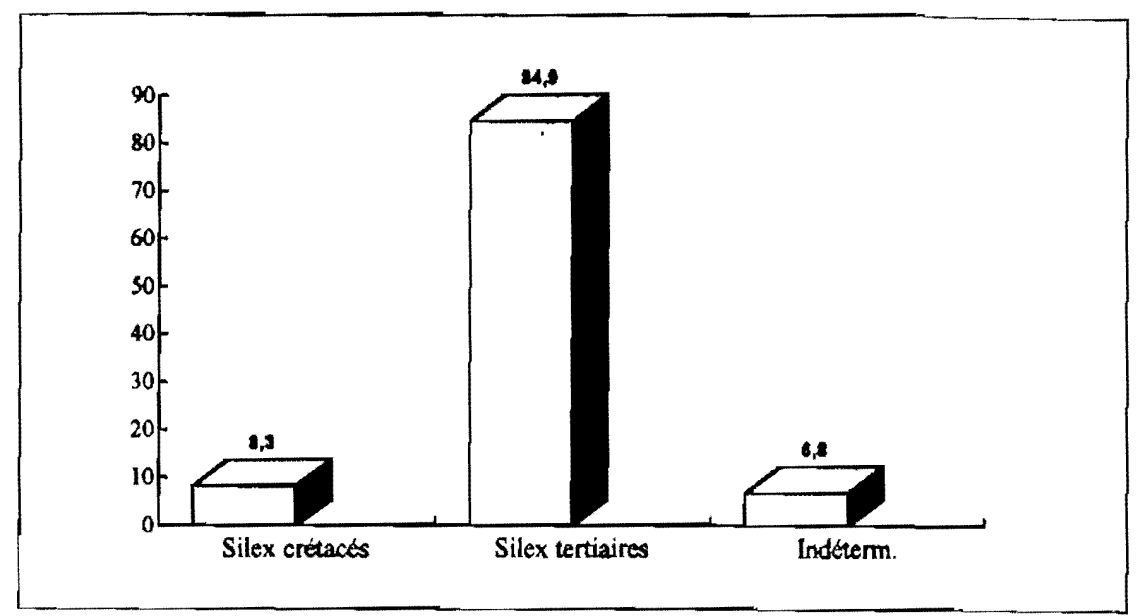

Fig. 2 - Répartition des différentes catégories de matières premières (en poids). taires (déterminations microfaciologiques P. Barrier, I.G.A.L.) de la Limagne et de ses marges.

Plusieurs sous-types peuvent être individualisés. Le plus abondant est un silex assez hétérogène, à grain assez grossier, souvent lité, de teinte jaune cire à marron très foncé. II est issu d'une formation calcaire oligocène de type palustre. Ce silex a été largement exploité par les préhistoriques de la région (Bourdelle et Virmont, 1983 ; Surmely, 1995 b). Le plus proche affleurement se trouve sur la commune de Laps, qui est distante d'Enval d'un kilomètre et demi. La matière première se présente sous la forme de bancs souvent massifs et souvent finement lités. Sa qualité est très inégale. Certains gros blocs sont fort bons et ont permis aux magdaléniens d'obtenir de très longues lames (Canque, Charvilhat et Goursonnet, 1929). On trouve aussi un silex noir à grain assez fin (brèche silicifiée d'origine volcano-sédimen- taire probable), qui provient des alentours (région de Saint-Maurice ou bien de Corent; Masson, 1981 ; Torti, 1980) et un silex de couleur jaune clair à marron clair, assez translucide, pouvant présenter des dendrites noires de manganèse (roche tertiaire silicifiée). La très grande variété de faciès des silex tertiaires rend particulièrement difficile une détermination précise de l'origine, par les méthodes traditionnelles (microfaciologie, pétrographie).

La seconde grande catégorie rassemble des silex très homogènes, translucides, à grain très fin et à cortex blanc, qui offrent deux variétés. La plus abondante est de couleur blonde. II y a aussi une variété de couleur gris foncé à noir, dont certains éléments sont assez proches du silex tertiaire noir. On retrouve ces silex sur tous les gisements préhistoriques de Limagne et du Velay, à partir du début du paléolithique su-

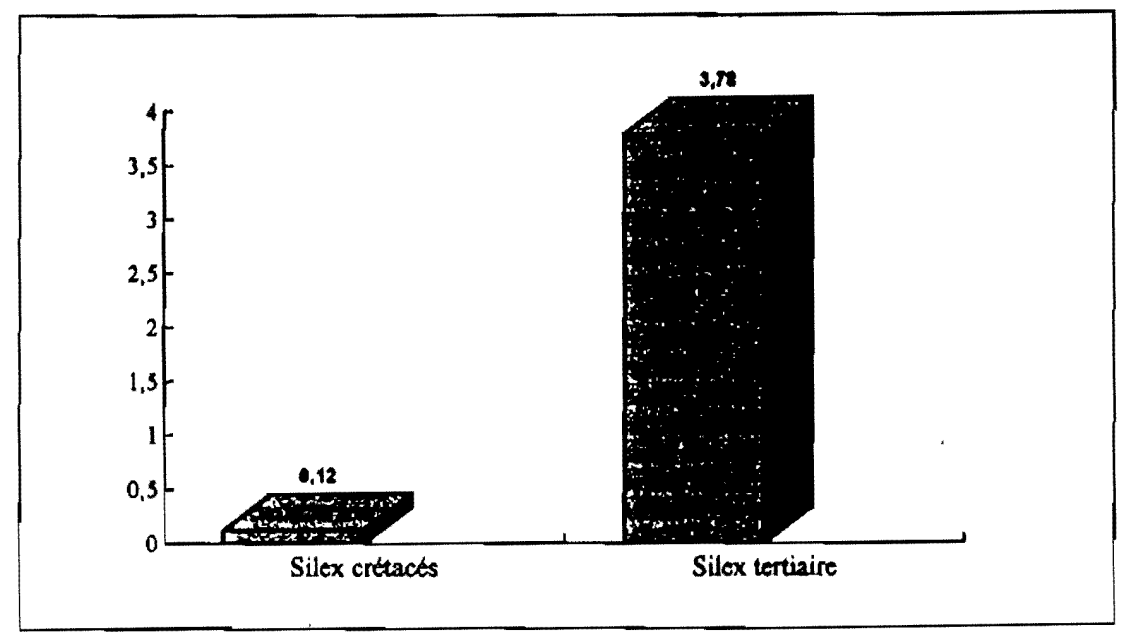

Fig. 3 - Rapport du poids déchets/supports pour chaque catégorie de matières premieres. 
périeur jusqu'au bronze moyen, avec des proportions variables selon les époques (Torti, 1980 ; Masson, 1981 ; Bourdelle et Virmont, 1983 ; Bracco, 1992 et 1994 ; Surmely et alii, à paraître). L'origine de cette matière première a été très discutèe (Masson, 1981 ; Torti, 1980). Les études pétroarchéologiques récentes confirment le diagnostic d'A. Masson, en montrant clairement que ces silex proviennent de formations calcaires marines du crétacé supérieur (Barrier, 1995 ; Surmely et al., à paraitre). Celles-ci sont totalement absentes en Auvergne et ne se rencontrent que dans le sud du Bassin Parisien (basses vallées de l'Indre et du Cher).

Enfin, quelques pièces s'individualisent de ces deux grandes catégories, et leur origine, probablement locale, est encore mal déterminée.

\section{- Le débitage}

Les deux grandes catégories de matières premières ont fait l'objet d'une gestion totalement différente (fig. 4) et qui apparaît clairement, malgré la modestie de l'échantillonnage de mobilier dont nous disposons.

Les silex tertiaires locaux sont représentés par un débitage abondant et volumineux, notamment pour le silex dit de "Laps". Toutes les étapes de la chaîne opératoire sont représentées, a l'exception cependant des blocs bruts. La faible représentation des déchets corticaux peut s'expliquer par la nature même du matériau, car les plaquettes sont le plus souvent dépourvues de cortex véritable. Le débitage a été effectué sur place, comme en témoigne la forte quantité de déchets abandonnés sur le site (fig. 3 et 4 ). L'abondance de ces derniers tient vraisemblablement à la qualité souvent médiocre et hétérogène de la matière première. Les nucléus abandonnés sur le site sont assez nombreux. Les 5 pièces découvertes se répartissent ainsi : un globuleux à plan de frappe préférentiel, deux prismatiques à lamelles (fig. $7, n^{\text {os }} 1$ et 2 ), un prismatique à lames et un débris.

Les silex tertiaires sont abondamment utilisés pour produire des supports variés, éclats, lames assez épaisses et lamelles, avec un taux de transformation relativement faible, sauf pour les lamelles. Toutefois, il faut envisager, pour les lames et éclats, la possibilité d'une utilisation du support

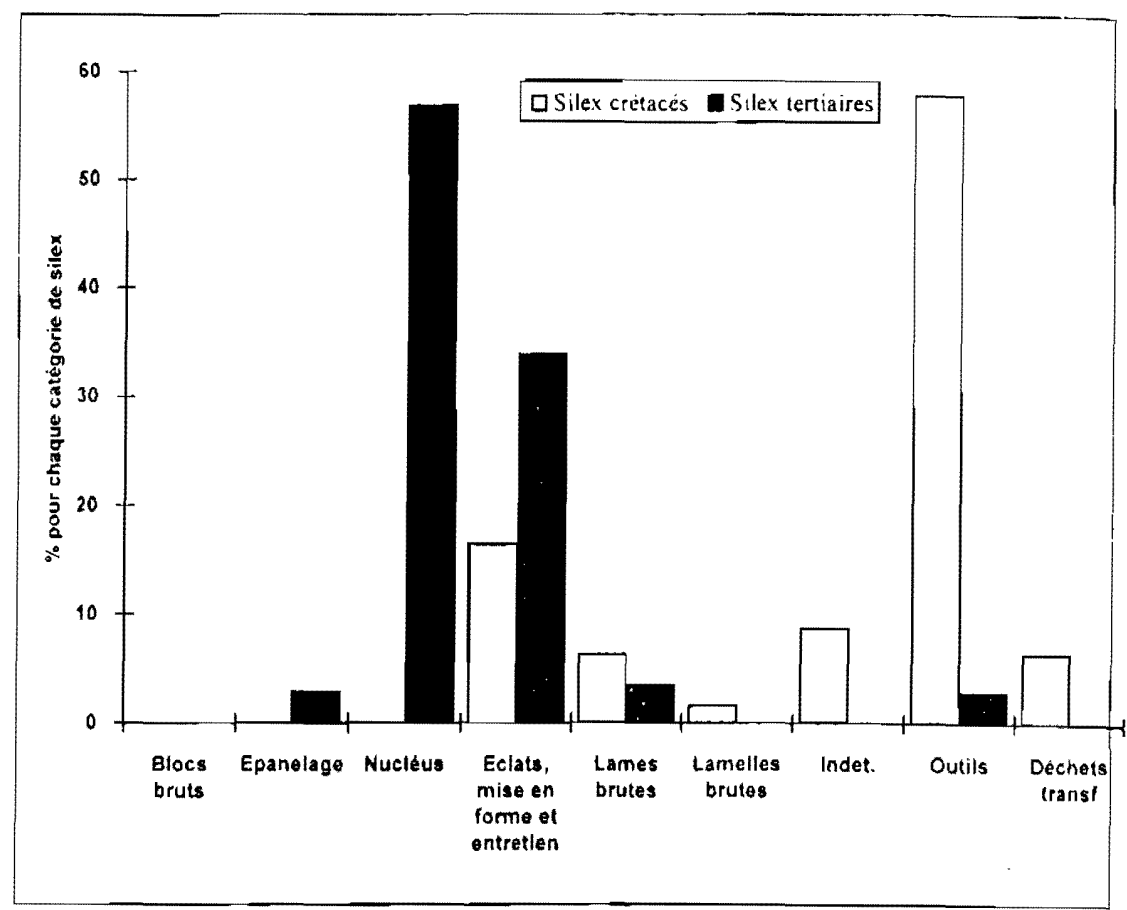

Fig. 4 - Répartition de l'industrie lithique par classes technologiques et catégories de matières premières (en poids et en pourcentage pour chaque type de matières premières).

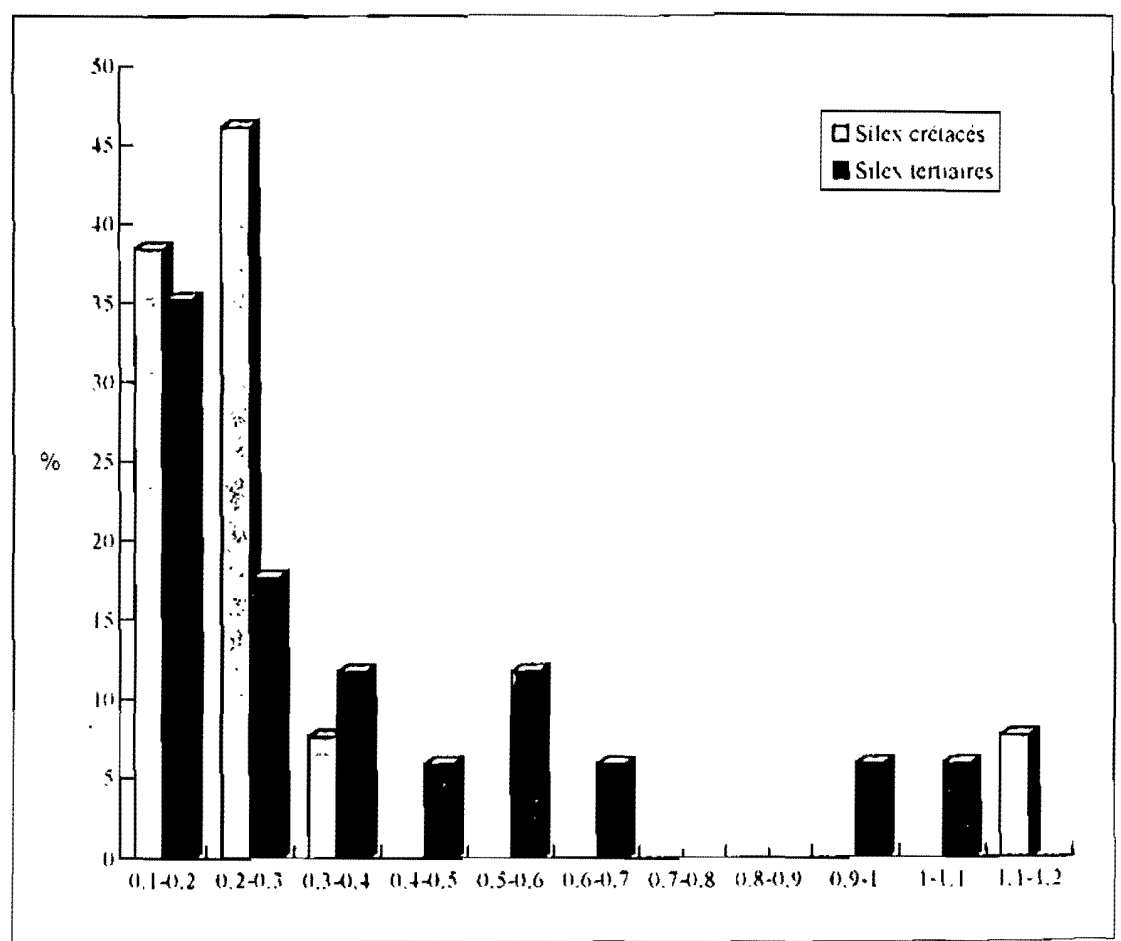

Fig. 5 - Répartition de l'épaisseur (en $\mathrm{cm}$ ) des supports laminaires et lamellaires, par catégories de matières premières.

brut, sans transformation. Seule une étude tracéologique complète permettrait de le confirmer. On peut également envisager la possibilité d'un emport de lames hors de l'abri.

Les silex crétacés (originaires du sud du Bassin Parisien), quant à eux, semblent avoir été introduits essentiellement sous la forme de nucléus déjà mis en forme et peut-être de supports préparés. La phase amont de la chaine opératoire n'est pas représentée et les déchets de mise en forme et d'entretien sont rares et de taille très réduite (fig. 4). Aucun nucléus n'a été découvert.

Le débitage des silex crétacés a été orienté vers la production de sup- 


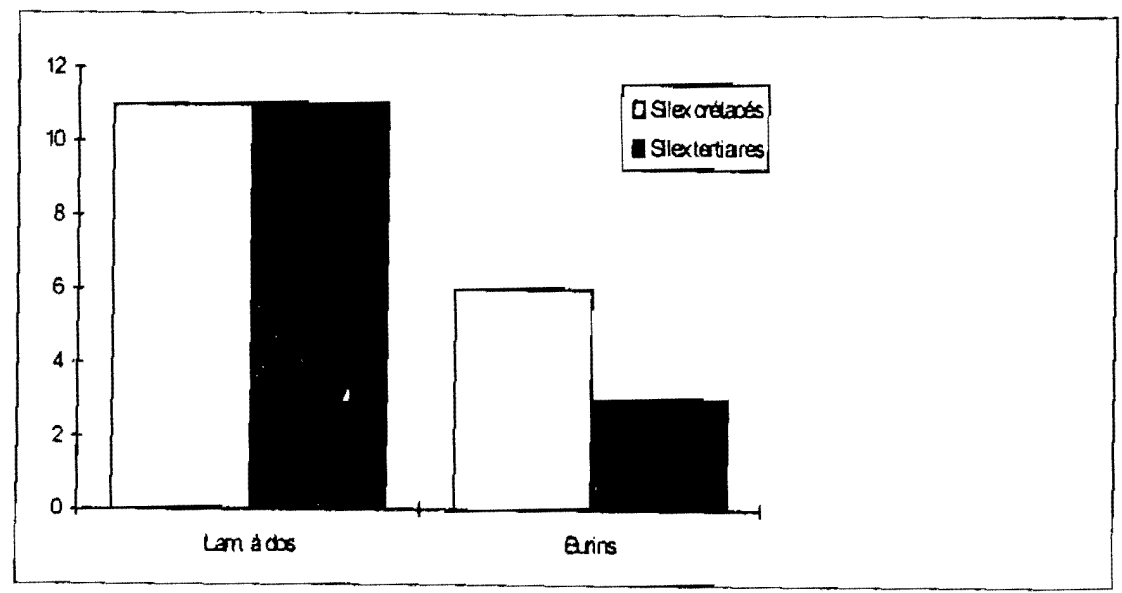

Fig, 6 - Répartition des outils, par catégories de matières premières.

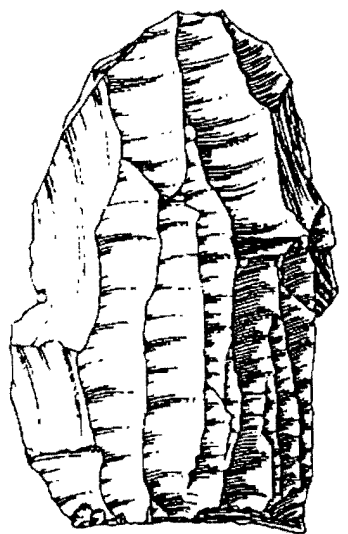

1

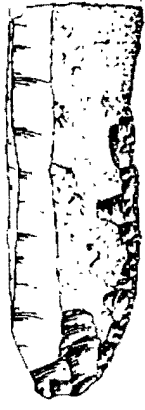

8

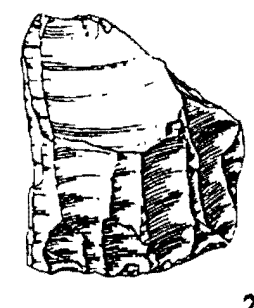

2

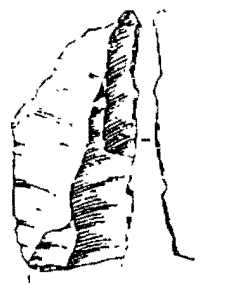

5
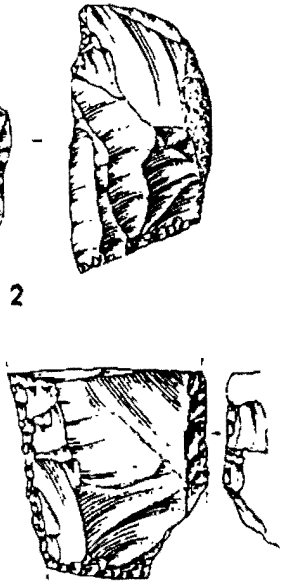

6
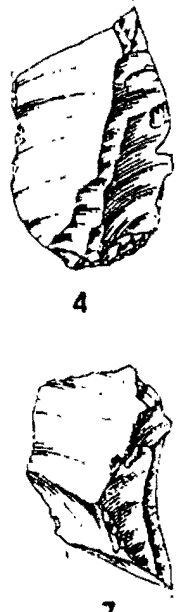

7

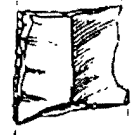

9

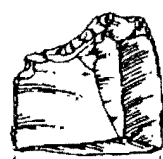

10

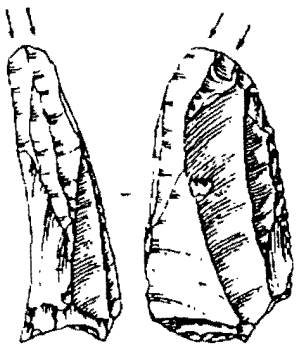

14
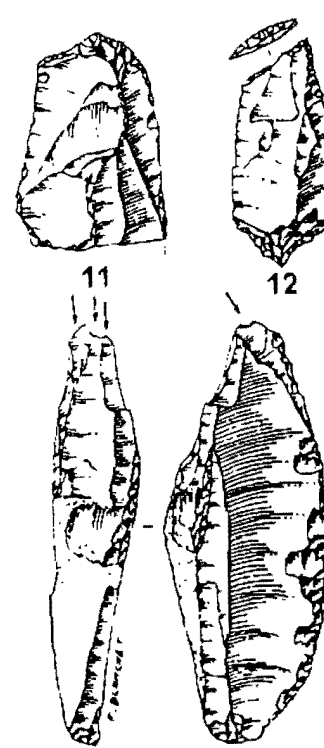

15

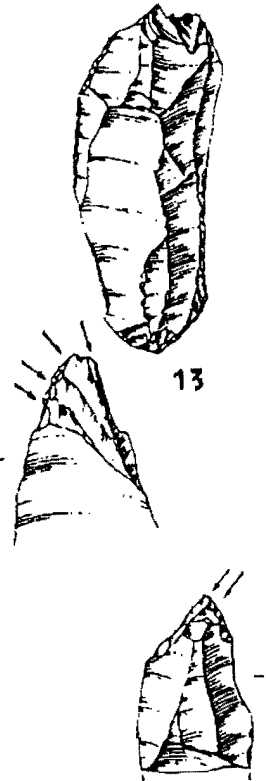

16

Fig. 7 - 1 et 2 : nucléus ; 5 : bec ; 8 et 14 à $16:$ burins ; 6,9 et 18 : lames retouchés ; 10 et 11 : grattoirs; 12 : percoir ; 13 : éclat retouché. Dessins F. Blanchet.

ports beaucoup plus fins que pour les silex tertiaires (fig. 5), notamment pour les lames. Ce mème caractère a été observé pour l'abri Durif (Torti, 1980). II est difficile de juger de l'indice d'allongement de celles-ci, en raison de la très forte réduction de des armatures dans le cas des lamelles.

Le taux de transformation en outils retouchés est très fort, ce qui révèle une utilisation optimale de la matière première. Le souci d'économie se traduit également par le ravivage fréquent et souvent multiple des burins (fig. 4).

Le recours aux silex crétacés exotiques peut s'expliquer par leur homogénéité, leur dureté et leur finesse de leur texture qui autorisent la pro. duction de lames élancées avec des tranchants acérés et très résis. tants (1) (particulièrement appréciables pour les burins). Le choix des silex crétacés pour la fabrication de burins dièdres apparaît clairement (fig. 6), alors que pour réaliser les lamelles à dos, dont le tranchant ne requiert pas les mêmes exigences, les magdaléniens ont eu recours indifféremment aux deux catégories de matière première. Cette sélection peut être constatée sur d'autres gisements magdaléniens régionaux, comme celui de Thônes. Elle a également été constatée pour d'autres types de matière première, comme le silex du Bergeracois, dans le nord de l'Aquitaine (Demars, 1994). Une étude tracéologique pourrait peutêtre même permettre de distinguer un usage spécifique réservé aux seuls burins façonnés en silex crétacés, mais il faudrait un échantillonnage plus large que celui dont nous disposons pour Enval 2.

\section{- L'outillage}

L'outillage retouché est dominé par les burins ( 8 ex.) et surtout par les armatures (22 ex.).

Ces dernières se répartissent selon les types suivants:

- 3 lamelles à dos simple, bitronquées (fig. $8, n^{\text {pos }} 5,6$ et 9 ) :

-8 fragments (dont 5 distaux) de lamelies à dos simple, tronquées (fig. $8, n^{\text {os }} 7,8,11,13-16$ et 20 ) :

- 1 fragment mésial de lamelles à dos double partiel (fig. $8, n^{\circ} 10$ ) ;

- 4 fragments de lamelles à dos simple (fig. $8, n^{\text {os }} 12,17 ; 18$ et 23 ) ; leur longueur par la transformation en outils fréquemment ravivés.

Ces supports de bonne qualité ont été transformés sur place pour obtenir des outils et notamment des burins dièdres sur lames, mais aussi
(1) Ce qui explique egalement que te mème silex at été préléré entré tous pour la tabrica. tion des pierres à fustl, à partir du XVIII siecle 
- 1 fragment de lamelle à retouche inverse partielle (fig. $8, \mathrm{n}^{\circ} 22$ );

- 2 petites pointes à dos double (dont 1 fragment distal) (fig. $8, n^{\infty} 19$ et 21);

- 3 fragments distaux de petites pointes à dos simple (fig. $8, n^{\circ 5} 24$ 26).

Au sein de cet ensemble, on peut individualiser deux groupes:

- un groupe de pièces à dos bien marqué et souvent double, façonnées sur des supports allongés, assez épais et fréquemment tronqués (fig. 8 , nos $5-9$ et $14-17$ ). Cette catégorie est la plus forte numériquement : un groupe comprenant des pièces à dos peu marqué, se limitant parfois à de simples retouches marginales, aménagées sur des lamelles plutót larges et à section fine.

L'existence de ces deux groupes a été observée également pour les lamelles à dos de l'abri Durif (Bourdelle et Merlet, 1991). II est a noter que ce phénomène ne saurait s'expliquer par la nature de la matière première, car les deux variétés de lamelles à dos se retrouvent pour tous les types de silex, mais plutôt par une spécificité fonctionnelle.

II faut également signaler la présence de petites pointes à dos courbe, déjà signalées dans des gisements auvergnats de la fin du magdalénien, comme celul de Cors (Surmely, 1995 a) :

-2 petites pointes à dos double (dont un fragment distal) (fig. $8, n^{\text {os }} 19$ et 21):

- 3 fragments distaux de petites pointes à dos simple (fig. 8, $n^{\infty}$ 24-26).

Parmi le reste de l'outillage, les burins sont nettement dominants, avec une forte représentation des exemplaires dièdres :

- 2 burins dièdres d'axe (fig. 7 , $n^{\circ} 16$ et $8, n^{\circ} 3$ );

- 1 burin d'angle brisé, sur lame retouchée (fig. $7, n^{\circ} 8$ );

- 1 burin d'angle sur troncature concave (fig. $8, n^{\circ} 2$ );

$\left.8, n^{\circ} 1\right)$;

- 1 burin caréné (fig. $\left.7, n^{\circ} 14\right)$;

-1 burin multiple (fig. $8, n^{\circ} 4$ ).
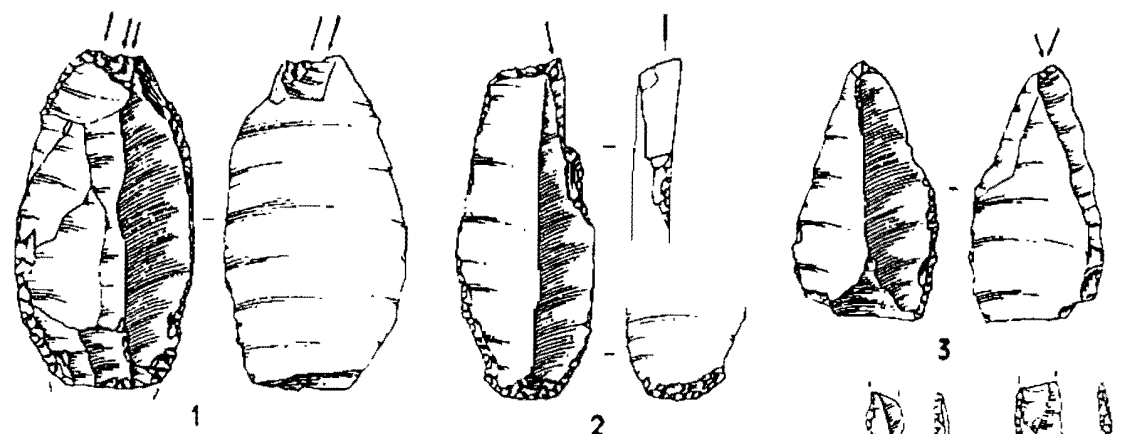

2
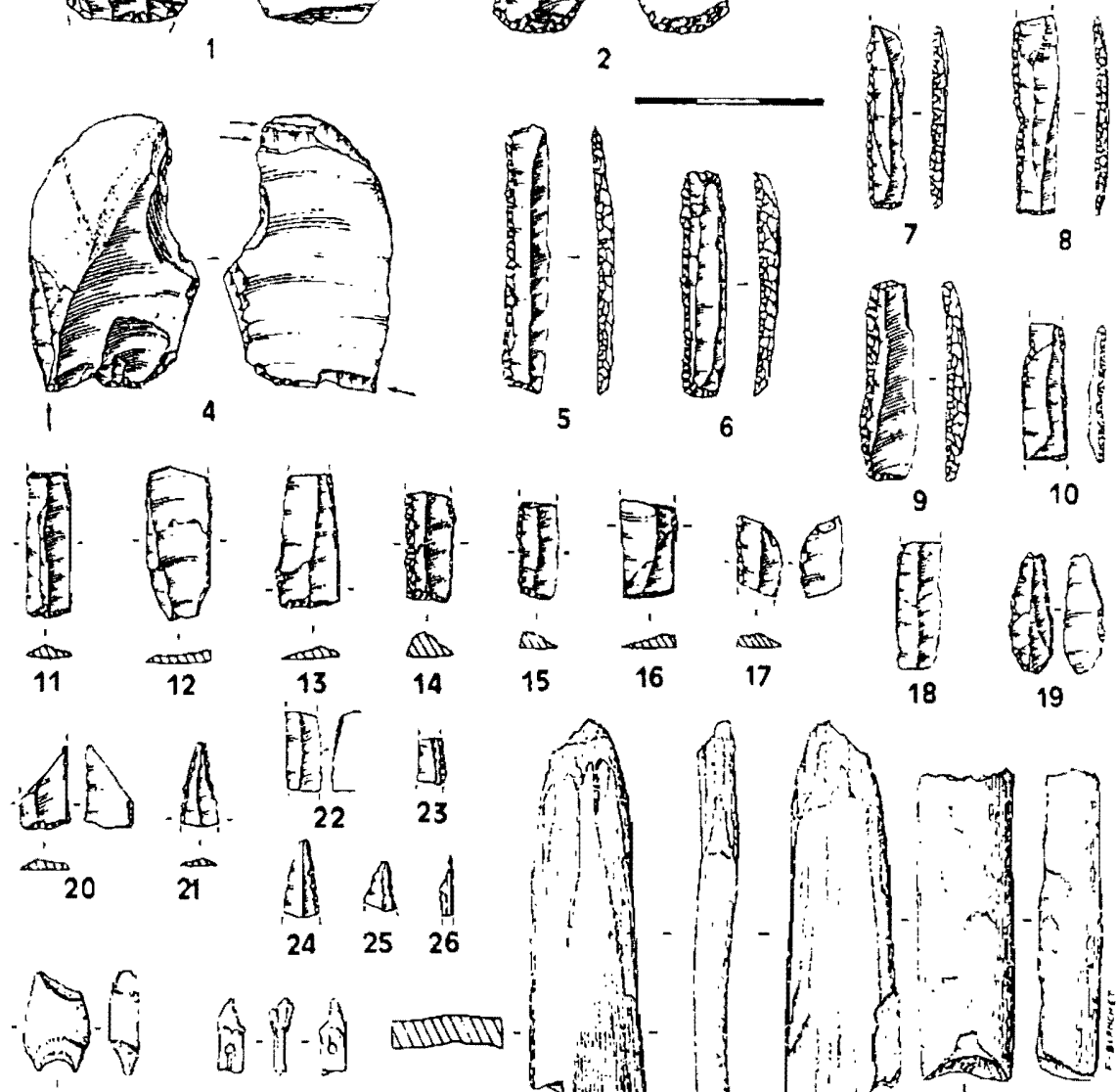

(III)

27

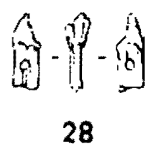

28

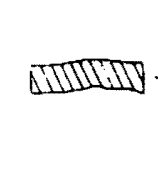

15

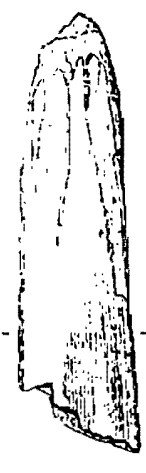

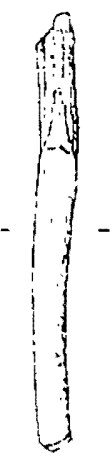

29

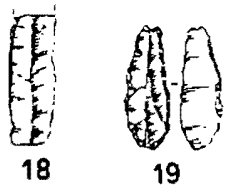

Fig. 8 - 1 à 4 : burins ; 5 à 26 : armatures ; 27 : incisive de boviné perforée ; 29 : lame en ivoire de mammouth ; 30 : fragment de baguette demi-ronde. Dessins F. Blanchet.

Le reste de l'outillage retouché se partage entre 3 lames retouchées (fig. $7, n^{\text {os }} 6,9$ et 18 ), 2 grattoirs ogivaux (fig. $7, n^{\circ} 10$ et 11), 1 bec (fig. $7, n^{\circ} 5$ ), 2 perçoirs, dont 1 double (fig. $7, n^{\circ} 12$ ), et 1 éclat retouché (fig. $7, n^{\circ} 13$ ).

En conclusion, les principales caractéristiques du gisement d'Enval 2 sont communes à la majorité des gisements magdaléniens de Limagne: prépondérance des burins et surtout des lamelles à dos au sein de l'outillage, utilisation prédominante des silex tertiaires locaux, utilisation plus réduite des silex crétacés d'origine lointaine.

Les caractéristiques propres à l'exploitation de ces deux grands types de matières premières s'expliquent fort bien par l'origine et les propriètés différentes de chacune des roches et montrent une gestion raisonnée et organisée de la matière première par les magdaléniens.

Reste, bien sûr, à s'interroger sur les modalités de transport des silex crétacés, dans ce secteur du Val d'Allier, à plus de 200 kilomètres de son lieu d'origine. Bien des hypotheses sont possibles (transport par le groupe lui-même au cours de migrations périodiques, voire saisonnières ; échanges entre groupes voisins...), mais elles demeurent à ce jour tout à fait conjoncturales (Bracco, 1992 ; Flébot-Augustins, 1994 ; Surmely, 1995 b). 


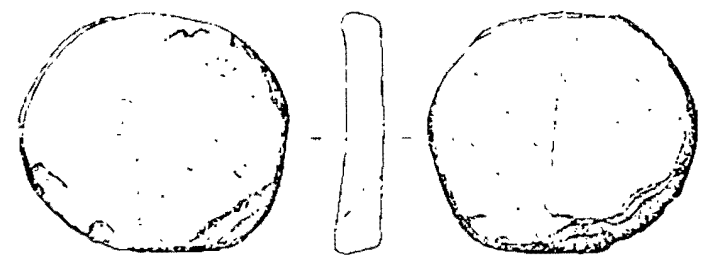

।

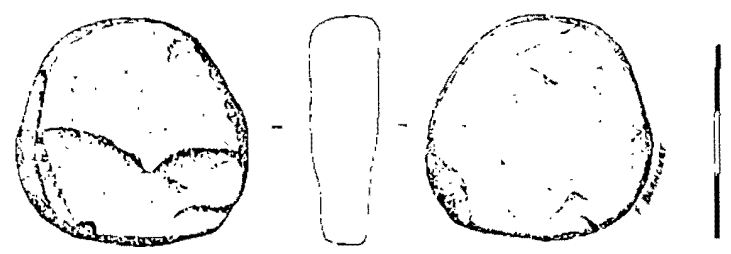

2

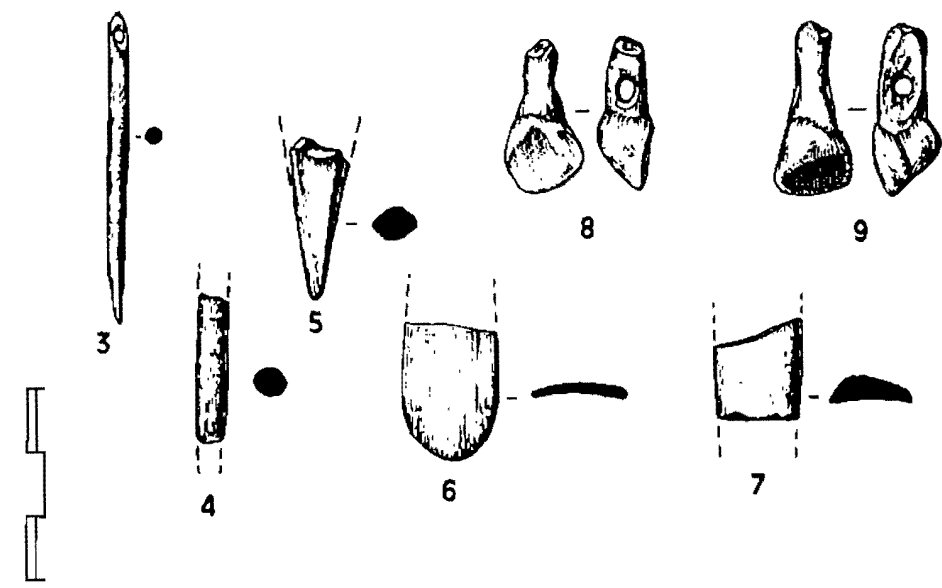

Fig. 9 - 1 et 2 : rondelles en gres ; 3 : alguille à chas ; $4:$ fragment de hampe de harpon en bois de renne; 5 : Alguille a chas en bols de renne; 6 : fragment de "lissoir"; 7 : fragment de bois de renne travaille ; 8 et 9 : incisives de bovinés perforées. Dessins F. Blanchet (1 et 2 ) et $A$. Urgal $(3$ à 7 ).

\section{L'INDUSTRIE OSSEUSE ET LES OBJETS DE PARURE (F.S. ET R.L.)}

Le mobilier osseux est assez abondant et présente un grand intérêt du fait de sa rareté générale en Auvergne, où les conditions de conservation sont souvent mauvaises (acidité ou oxydation).

II faut noter la présence d'un fragment de base de harpon (fig. $9, n^{\circ} 4$ ), d'une aiguille à chas complète (fig. 9 , $n^{\circ} 3$ ), d'une extrémité distale de sagaie en bois de renne (fig. $9, n^{\circ} 5$ ), de trois fragments de baguettes demirondes (fig. $8, n^{\circ} 30$ ), de trois incisives de bovinés percées (fig. 8 , $n^{\circ} 27$ et fig. $9, n^{\infty} 8$ et 9 ) dont l'extrémité inférieure a été tronquée et aplanie (Taborin, 1970), d'un fragment de lissoir (fig. $9, n^{\circ} 6$ ) et d'une autre pièce en bois de renne (fig. $9, n^{\circ} 7$ ).

Parmi les pièces singulières on trouve :
- une lame en ivoire de mammouth (fig. 8, n०29). C'est un fragment mince et allongé $(112 \mathrm{~mm})$, tiré d'une défense, dont les bords ont été soigneusement régularisés ;

-3 rondelles en grès (substrat rocheux). Ces rondelles, non perforées, présentent une forme grossièrement circulaire, deux faces lisses et des bords aux angles adoucis.

La plus grande a un diametre de $36 \mathrm{~mm}$ et une épaisseur de $9 \mathrm{~mm}$ (fig. $9, n^{\circ} 1$ ). La seconde a un diamètre de $36 \mathrm{~mm}$ et 6 d'épaisseur (fig. $9, n^{\circ} 2$ ). La troisième est cassée (diamètre 22, épaisseur 2,5). L'abri Durif avait livré deux fragments de rondelles, dont une en grès (Bourdelle, 1987). Cette roche a été aussi utilisée pour les plaquettes gravées, certainement en raison de sa faible dureté qui facilite son travail. Mais la rondelle en grès découverte dans l'abri Durif est mince $(2,5 \mathrm{~mm})$, perforée, décorée de traits gravés et constitue à coup sûr un élément de parure. Des rondelles non perforées minérales ont déjà été signalées dans le magdalénien pyrénéen ( $\mathrm{Ba}$ randiaran, 1968 ; Sieveking, 1971).

Les trois rondelles du gisement d'Enval pourraient être des produits semi-finis. Cette hypothèse est particulièrement vraisemblable pour la pièce cassée la plus fine, qui aurait pu être brisée lors de sa fabrication. avant sa perforation. Une autre fonction, encore indéterminée, n'est toutefois pas à exclure, notamment pour les deux rondelles les plus massives.

II faut signaler l'absence totale d'œuvres d'art mobilières (pourtant nombreuses à l'abri Durif). Le gisement d'Enval 2 a livré cependant un petit crayon d'ocre, portant des traces très nettes d'usure par abrasion.

\section{LA FAUNE (L.F.)}

Les restes osseux et dentaires des sites paléolithiques étant rarement conservés en Auvergne, la faune d'Enval 2 n'est pas dénuée d'intérêt, même si cet échantillon ne compte que 1091 restes au total. Sa représentativité est cependant probablement assez faible (cf. supra) et les conclusions relatives à la saisonnalité de la chasse et à sa spécialisation devront être considérées avec prudence.

\section{L'état du matériel et la détermination}

L'état de conservation de ce matériel est correct, puisque de nombreux restes de petite taille ou particulièrement fragiles ont été conservés : métapodes vestigiaux d'un fœtus de Renne, bourgeons dentaires d'une incisive déciduale de boviné et d'une prémolaire définitive de Renne, prémolaire déciduale de Chamois, restes de Lapin.

Le matériel est très fragmenté : seulement treize pièces sont entières et, sur le total de fragments osseux (1 091), plus de la moitié (552) sont des esquilles de moins de deux centimètres. En raison de ce fort degré de fragmentation, qui ne surprend pas dans un contexte magdalénien et particulièrement dans celui-ci (cf. supra), la détermination a été délicate : seuls 85 restes ont été déterminés spécifiquement et anatomiquement. Parmi les restes indétermi- 
nés, certains peuvent appartenir au Renne, d'autres à des herbivores de plus grande taille, comme les équidés et les grands bovinés, ce que nous avons précisé.

\section{- Les espèces représentées} (fig. 10)

Le Renne (Rangifer tarandus) domine l'assemblage en nombre de restes. Les deux seuls fragments de bois mis au jour sont deux fragments de pointe d'andouiller, appartenant probablement à un individu adulte.

Le Cheval (Equus caballus) est représenté par treize restes déterminés anatomiquement : quatre fragments de mandibule, sept fragments de dents et deux fragments de première phalange.

II est difficile d'identifier l'espèce en l'absence de restes entiers mesurables, même si, à la fin du Würm, il s'agit souvent d'Equus caballus (Prat, 1968).

Les bovinés (Bison priscus, Bos primigenus) sont attestés par trois restes déterminés : un sésamoïde, une première phalange fragmentée et un bourgeon d'incisive déciduale.

Nous n'avons pas pu préciser le genre représenté, Bos ou Bison, à partir de ces seuls restes.

Le Chamois (Rupicapra rupicapra) est représenté par une seule seconde prémolaire inférieure.

Le Cerf (Cervus elaphus) est représenté par un fragment de bois, plus précisément une pointe d'andouiller appartenant à un individu adulte.

Le Renard Nulpes vulpes, Alopex lagopus) est le seul carnivore attesté, représenté par deux restes : une canine supérieure et un fragment proximal de radius. Ils ne permettent pas de préciser s'il s'agit du Renard commun ou du Renard polaire, car ce ne sont pas des éléments anatomiques discriminants (Poplin, 1972).

Le Lapin (Oryctolagus cuniculus) est représenté par deux extrémités distales de première phalange et de métapode. II est intéressant de noter sa présence dans cette région, car, même si ses restes sont présents dans quelques sites de la HauteLoire, ils se situent plus souvent au sud du Massif Central. Leur carac-

\begin{tabular}{|c|c|c|}
\hline Espèces & NR & NMIf \\
\hline Rempgifer taramulus & 63 & 3 \\
\hline Indét. of Rangifer laramuliss' & 698 & 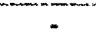 \\
\hline Expuns ( cothallus & 13 & 1 \\
\hline Bos BIsom & 3 & 2 \\
\hline Indet. cf. Fiquas Bos Bisom & 57 & - \\
\hline ("erv'ls likphluss & $\hat{i}$ & 1 \\
\hline Rupricapra rilpicapret & $i$ & 1 \\
\hline liilpes iwlpes Alopex lagopus & 2 & 1 \\
\hline Oryctolagus cumiculas & 2 & 1 \\
\hline Oiseau & 1 & $i$ \\
\hline Frag d'os brülés indet & 250 & 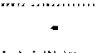 \\
\hline Total déterminés & 85 & - \\
\hline Total indetermines & 1006 & $=$ \\
\hline TOTAL & 1091 & 11 \\
\hline
\end{tabular}

Fig. 10 - Espèces animales représentées.

tère intrusif ne peut être exclu, surtout dans le contexte archéologique d'Enval 2 (cf. supra).

\section{- Chasse et consommation}

Toutes les espèces déterminées ont-elles été chassées par l'homme, ou bien l'origine de certaines est-elle différente ? L'étude de la fragmentation, l'observation des traces de découpe et la fréquence des parties du squelette permettent souvent de distinguer des restes intrusifs d'animaux dont l'origine est la mort naturelle, ou qui ont été la proie de carnivores ou de rapaces. A Enval 2 . la question est délicate en raison du faible nombre de restes (six) de Cerf, de Chamois, de Renard et de Lapin, d'autant qu'ils ne portent visiblement aucune trace de découpe. Le caractère intrusif des carnivores ou du Lapin est souvent discuté (David, 1994 ; Fullola et al., à paraître), mais il est impossible de trancher. On peut seulement constater qu'aucune trace de la présence et de l'action de carnivores n'a été observée.

Les restes de Cheval et de boviné déterminés anatomiquement ne portent aucune trace. Cependant, l'observation des stries sur les os des grands herbivores a été un exercice vain dans la moitié des cas : les restes osseux sont couverts de larges plages d'oxyde de manganèse qui rendent invisibles les éventuelles traces. Deux d'entre elles ont pu toutefois être observées sur deux restes indéterminés de bovinés/équidés. De plus, la fragmentation de ces restes est typique d'une fracturation anthropique (Patou, 1994).
Parmi les restes de Renne déterminés anatomiquement, quatre d'entre eux seulement portent des traces de découpe, toujours en rapport avec l'état de surface.

La nature de l'échantillon rend périlleuse la tentative d'analyse de la représentation des parties osseuses (fig. 11). Toutes les parties du squelette sont représentées, à l'exception des ceintures scapulaire et pelvienne. Cette absence peut difficilement s'expliquer par le jeu de la conservation différentielle (ce ne sont pas les parties les moins résistantes du squelette), mais plutôt par la détermination différentielle et surtout l'échantillonnage, tout comme la forte représentation des rotules. II est donc probable que certains rennes aient été apportés entiers sur le site.

\section{- Saisonnalité de la chasse}

Les Magdaléniens ont donc chassé principalement le Renne, le Cheval, et l'Aurochs ou le Bison, mais ce tableau de chasse est probablement incomplet, en raison de la petite taille de l'échantillon et de sa faible représentativité. Si l'herbivore majoritairement chassé peut être le renne, on ne peut pas préciser le type de chasse, en l'absence de restes dentaires permettant d'identifier l'áge des animaux tués.

Cependant, ces restes nous livrent une information fondamentale, directement liée à notre probléma. tique, qui est celle de la saisonnalité de l'occupation.

Les données disponibles pour le site d'Enval 2 semblent montrer que 


\begin{tabular}{|l|c|c|}
\hline \multicolumn{1}{|c|}{ Parties squelettiques } & NR & NMPS \\
\hline Bois & 2 & 1 \\
\hline Dents jugales supérieures & 2 & 1 \\
\hline Dents jugales inférieures & 2 & 2 \\
\hline Mandibule & 4 & 3 \\
\hline Humérus & 2 & 1 \\
\hline Radius & 1 & 1 \\
\hline Métacarpe & 1 & 1 \\
\hline Carpe (pisiforme et scaphoìdes) & 3 & 3 \\
\hline Côtes & 9 & 3 \\
\hline Vertèbre (lombaire) & 1 & 1 \\
\hline Fémur & 3 & 2 \\
\hline Patella & 3 & 3 \\
\hline Tibia & 1 & 1 \\
\hline Maléole tibiale & 1 & 1 \\
\hline Tarse (talus) & 2 & 2 \\
\hline Métatarse & 6 & 4 \\
\hline Métapode indét. & 5 & - \\
\hline Métapode vestigial & 2 & 2 \\
\hline Phalange I & 3 & 3 \\
\hline Phalange II & 3 & 3 \\
\hline Phalange III & 2 & 2 \\
\hline Phalange vest. I & 2 & 2 \\
\hline Phalange vest. IIl & 1 & 1 \\
\hline Sésamoïdes & 2 & 2 \\
\hline TOTAL & 63 & 45 \\
\hline & & \\
\hline
\end{tabular}

Fig. 11 - Parties squelettiques du Renne.

les animaux ont été chassés à un seul moment de l'année, entre les mois de février et juin. En effet, les restes d'un fcetus de Renne, d'un Chamois et d'un boviné de la première année, montrent, après la détermination de leur áge respectif, qu'ils ont été abattus au printemps et peut-être dès la fin de l'hiver (si le boviné en question est un Bison).

Ces données ne sont établies qu'à partir de trois restes, mais ils produisent tous le même résultat. De plus, elles concernent trois especes différentes, ce qui tendrait à plaider en faveur de l'occupation du site à une seule saison de l'année.

Quels résultats cette étude de la faune d'Enval 2 a-t-elle apportés ?

Tout d'abord, les espèces identifiées forment un tableau de chasse tout à fait comparable à ce que l'on peut observer dans les sites magdaléniens des Pyrénées (Clottes, 1987 ; Clot, 1984 et 1988), de l'Aude (Sacchi, 1986 ; Fontana, 1995), du SudOuest, ou même du reste de l'Europe (Delpech, 1989 et 1992). Cette conclusion s'applique également à l'Auvergne (Fontana, à paraître), mais on doit constater que dans l'abri Durif, F. Delpech a identifié l'Antilope saïga et le Bouquetin. dont nous n'avons pas trouvé trace à Enval 2.
Le résultat le plus important reste la mise en évidence d'une saison d'occupation de ce site (printemps et peut-être fin de ('hiver).

Mais cet ensemble à lui seul ne permet pas de confirmer l'attribution chronologique au magdalénien final (cf. supra), en l'absence d'espèces caractéristiques de certaines conditions climatiques, et d'os entiers mesurables.

En réalité, cet échantillon est caractéristique, par sa taille modeste et sa représentativité faible, des collections fauniques des sites magdaléniens de l'Auvergne. Les fouilles anciennes n'ont pas toujours récolté les restes fauniques et les investigations actuelles sont de simples sondages. Ces derniers ne livrent pas la quantité de matériel suffisante pour déterminer toutes les espèces chassées et analyser les restes osseux sous l'angle des modalités d'acquisition et des types de prédation. Sans des données plus substantielles, les sites magdaléniens ne pourront être caractérisés avec précision, et les modalités d'occupation du territoire ainsi que leur évolution resteront $\mathrm{mal}$ connues. A cet égard, l'étude (en cours) des restes fauniques des niveaux magdaléniens du Blot (HauteLoire) devrait apporter de nouveaux éléments.

\section{- La présence du mammouth} (F.S., R.L.)

Comme nous l'avons vu plus haut, une lamelle en ivoire de mammouth a été découverte sur le site. L'abri Durif avait déjà livré une molaire de cet animal. Ces éléments isolés ne résultent probablement pas d'un abattage, mais plutôt de carcasses ou éléments de carcasses découverts par les magdaléniens sur les rives de l'Allier (aujourd'hul encore, des éléments osseux de mammouths y sont découverts périodiquement ; Surmely, 1993) et rapportés au campement. De ce fait, on ne saurait considérer la présence de ces pieces comme la preuve d'une stricte contemporainéité de l'occupation avec la présence du proboscidien.

\section{LES RESTES HUMAINS \\ (L.F.)}

Le gisement a livré une molaire humaine. Notons qu'une molaire lactéale avait déjà été découverte dans l'abri Durif (Bourdelle, 1987) et qu'un squelette humain aurait été exhumé anciennement dans l'un des abris (Biélawski, 1887). Cette dent est affectée d'une carie. Nous n'avons toutefois pas la certitude absolue qu'elle soit bien contemporaine des autres vestiges.

\section{CONCLUSION (F.S., L.F.)}

Le gisement d'Enval 2, malgré les carences liées aux conditions de découverte et à la faiblesse quantitative des vestiges, présente un intérêt certain pour la caractérisation de l'occupation du Val d'Allier à l'époque magdalénienne.

L'ensemble du mobilier lithique et osseux, malgré le petit nombre de pièces, permet de dater l'occupation de la fin du magdalénien. L'industrie présente des similitudes évidentes avec celle découverte dans l'abri Durif et plus particulièrement le "Sol de la Grange". L'occupation de l'abri d'Enval 2 pourrait donc être datée de 13000 BP environ (2).

(2) Une datation absolue effectuée sur des esquilles osseuses a donne 10170 BP. Nous considerons que cette date ne peut être retenue et témoigne d'une pollution vraisemblable do l'echantillon par un fragment osseux olus réc l'bcht cent. Rappelons en our qu'une date aberante a été obtenue pour le Sol de la Grange de l'abri Durit (Bourdelle. 1987). 
Mais surtout, l'étude du gisement d'Enval 2 permet de confirmer l'importance de l'occupation magdalénienne du site d'Enval, qui se manifeste par la densité de l'habitat, l'abondance des vestiges et la présence de nombreux objets d'art et de parure. Autant de critères qui marquent la différence d'Enval avec les petits gisements de la moyenne montagne proche et plaident en faveur d'un grand campement, fréquenté de façon régulière. L'attraction qu'a pu provoquer le site sur les populations magdaléniennes, tient certainement, nous l'avons déjà vu, à la conjonction de facteurs favorables : proximité de sources importantes de matières premières, présence d'un escarpement tourné vers le sud et creusé de cavités, contexte géographique assurant l'accès à des niches écologiques variées.

Il est bien évidemment difficile, compte tenu de l'importance des mutilations subies par le site, de caractériser précisément l'occupation et notamment son organisation spatiale. L'abri Durif et Enval 2 ont été fouillés sur des surfaces très réduites et nous ignorons encore les relations exactes qui unissalent ces deux emplacements, distants de moins de $50 \mathrm{~m}$.

Même si certains rennes ont été apportés entiers sur le site, les échantillons ne sont pas assez représentatifs pour conclure que les gibiers y étaient systématiquement dépecés. Le renne semble avoir été une proie de prédilection, mais sa part exacte dans l'alimentation reste à définir. Enfin, même si nous savons qu'Enval 2 a été occupé très certainement au printemps, il est difficile de préciser la durée du séjour des hommes.

Les relations qui pouvaient unir Enval aux autres gisements régionaux restent également à déterminer. II faut noter qu'à proximité immédiate d'Enval, au débouché de la vallée sur la rivière Allier, se trouvent d'importants gisements de plein-air qui paraissent contemporains et livrent surtout des restes de chevaux (Charvilhat, 1911 ; Perreau, 1943 ; Bourdelle et Virmont, 1983 ; Surmely, 1995 ; Fontana, à paraitre). On peut donc envisager un lien de complémentarité, éventuellement saisonnier, entre ces deux concentrations d'habitats d'un type different. Plus généralement, on peut se demander quel type de relations pouvait exister entre les gisements de Limagne et les petits sites magdaléniens qui sont connus dans la moyenne montagne proche (Surmely, 1995 b).

L'hypothese d'une circulation plus étendue encore des groupes humains à travers le Val d'Allier reste également envisageable (Bracco, 1992 ; Fontana, a paraitre). L'utilisation de silex d'origine lointaine tendrait à indiquer la grande mobilité des magdaléniens, mais cette hypothèse reste subordonnée à la détermination du mode d'acquisition de ce matériau.

Les études archéologiques sont encore à ce jour trop peu avancées pour permettre de caractériser les modalités du peuplement de la Limagne et de ses marges au tardiglaciaire. Mais cette question constitue désormais pour nous une problématique de recherche.

\section{Remerciements}

Nous adressons nos plus vifs remerciements à $M$. François Blanchet qui a assuré gracieusement l'illustration de cet article.

\section{Bibllographie}

BarRIER P. (1995) - Expertiso microfaciologique de silex d'intérét archéologique (S.R.A. Auvergne). Cergy-Pontoise, rapport REIG $n^{\circ}$ 95-146, $13 p$. 17 pl., dact.

Baudon Th. (1911) - Des lampes en roches dures, en pierre ollaire et en craie fabriquées par l'Homme aux époques paléolithique, néolithique et pendant l'áge du bronze. Bulletin de la Société historique et scientifique de I'Oise, p. 115-159.

BielaWsKI J.-B. (1887) - Récits d'un touriste auvergnat, Issoire, p. 285.

Boufdelle Y. (1977) - L'abri Durif à Enval (commune de Vic-le-Comte, 63). In : La fin des temps glaciaires en Europe. Bordeaux, C.N.R.S., p. 389-398.

Boundelle Y. (1987) - Rapport de synthese des foullies d'Enval de 1969 a 1987. Clermont-Ferrand, direction des Antlquités d'Auvergne, dact.

Bourdelle Y. et Daugas J.-P. (1969) Premierres observations sur l'industrie du gisement magdalénien d'Enval (commune de Vic-le-Comte), Congrès préhistorique de France, XiXtome session, p. 107-114.

Bourdelle Y., Delporte H. at Virmont J. (1971) - Le gisement magdalénien et la Venus d'Enval, L'Anthropologie, t. 75, p. 119-128.

Bourdelle Y. et Maziere G. (1983) L'art préhistorique. In : Les Inédits de la préhistoire auvergnate. ClermontFerrand, musee Bargoin, p. 163-183.
Boufdelle $Y$, et Merlet J.-C. (1987) Nouvelles découvertes d'art mobilier à Enval (Puy-de-Dóme). In : L'art des objets au paleolithique supérieur. Tome 1 : L'art mobilier et son contexte. Paris, éditions du C.T.H.S., p. 255-259.

Bourdelle Y. el Merlet J.-C. (1991) - Le site d'Enval, commune de Vic-leComte (Puy-de-Dóme). Bulletin de la Société Préhistorique Française, t. 88 , p. 109-113.

Boufdelle Y. et Virmont J. (1983) - Le magdalénien des Limagnes et leurs bordures. In : Les Inédits de la préhistoire auvergnate. Clermont-Ferrand, musée Bargoin, p. 153-162.

Candue A., Charvilhat G. et GoursonNET M. (1929) - Un abri d'epoque magdalénienne à Enval. Revue d'Auvergne, t. 43, p. 65-71.

Chapmimat G. (1911) - Fouille d la station magdalénienne du Pont-de-Longues, prés la gare de Vic-le-Comte (63). Bulletin de la Société Próhistorique Francaise, t. 8, p. 777-778.

Clor A. (1984) - Faune de la grotte préhistorique du bois du Cantet (Espèche, Hautes-Pyrénées, France), Munibe, 36, p. 33-50.

Clor A. (1988) - Faune magdalénienne de la grande grotte de Labastide (Hautes-Pyrénées, France), Munibe, 40, p. 21-44.

Clotres J. (1989) - Le Magdalénien des Pyrénées. In : Le Magdalénien en Europe, actes du colloque de Mayence. Liege, ERAUL, 38, p. 281-360.

Daniel P. (1979) -- La station magdalénienne du Sire à Mirefleurs (Puy-deDóme). In : La fin des temps glaciaires en Europe. Bordeaux, C.N.R.S., p. 535-536.

DAVID F. (1994) - L'action des camivores dans les gisements pléistocènes d'Europe. Rappel de quelques étapes de la recherche en Europe occidentale. In : Outillage peu élaboré en os et bois de cervides, 6 table ronde : taphonomie. Paris, p. 77-82.

DELPECH F. (1989) - L'environnement animal des Magdaléniens, in Le Magdalénien en Europe, actes du colloque de Mayence. Liege, ERAUL, 38, p. 5-30.

DELPECH F. (1992) - Le monde magdalénien d'après le milieu animal. In : Le peuplement magdalénien. Paléogéographie physique et humaine, actes du Colloque de Chancelade. Paris, editions du C.T.H.S., p. 127-136.

Delpopte H. (1974) - Les Martres de Veyre-Le Bay. Gallia Préhistoire, t. 17, fasc. 2, p. 611 .

Demars P.-Y. (1994) - L'économie du silex au paleolithique superieur dans le nord de l'Aquitaine. Thèse d'État de I'université de Bordeaux I. Bordeaux, 2 vol, 549 et 270 p. dact.

Esteve N. (1942) - Éfude des accidents siliceux des calcaires tertiaires de la 
Limagne. Revue des Sciences naturelles de l'Auvergne, vol. 8, p. 30-56.

Flebor-Augustnos J. (1994) - La circulation des matieres promieres lithiques au paleolithique. Synthèse des données, perspectives comportementales. Thèse de doctorat, Université de $\mathrm{Pa}$ ris X-Nanterre, $477 \mathrm{p}$. dact.

FONTANA L. (1995) - Chasseurs magdaleniens et rennes en Bassin de l'Aude : analyse préliminaire, Anthropozoologica, 21, p. 147-156.

Fontana L. (1996) - Etat de la recherche et problématiques en archéozoologie pour le Paléolithique supérieur d'Auvergne, Cahiers archéologiques de Bourgogne, $n^{\circ} 6$, p. 00.

Fullola et alii (à paraître) - Le Paléolithique supérieur méditerranéen ibérique : approches paléenvironnementales, industrielles et artistiques. In : Les faciès leptolithiques du Bassin mo diterranéen nord-occidental: milieux naturels et culturels, XXVI congres prénistorique de France, Carcassonne. 1994.

Masson A. (1981) - Pétroarchéologie des roches siticeuses, intérét en préhistoire. Thèse de troisième cycle, Université de Lyon I.

PALEs L (1979) - L'abri Durif à Enval, gravures et sculptures sur pierre, GalliaPrehistoire, t. 22, fasc. 1, p. 113-142.

Patou M. (1994) - Actions anthropiques sur les assemblages osseux et sur les ossements du Paléolithique : état des recherches. In : Outillage peu elaboré en os et bois de cervidés, 6" table ronde : taphonomie. Paris, p. 133-142.
Perpere M. (1979) - Grotte Auzary a Thónes-le-Vieux, commune de Grandeyrolles (Puy-de-Dóme). In : La fin des temps glaciaires en Europe. Bordeaux, C.N.R.S., p. 569-570.

Perpere M. (1981) - Le magdalénien final de la grotte Auzary (Puy-de-Dóme), Bulletin de la Société Próhistorique Française, 1. 78 , fasc. 10.12 , p. 342 . 353.

Perreau A. (1943) - L'âge du Renne en Auvergne dans le bassin de l'Allier, Revue des Sciences Naturelles de l'Auvergne, t. 9, fasc. 1-4, p. 87-116.

POPUN F. (1972) - Contribution à la morphologie et à la biométrie d'Alopex lagopus (Linné) et de Vulpes vulpes (Linné) : Les Renards d'Arcy-sur-Cure. Thèse de doctorat de IIP cycle, Université de Paris VI.

Raynal J.-P. Vernet G. Fain J., Mialuer D., Montaet M., Pilleyre Th., Sanzelle S. et Davans J..P. (1994) - Téphrostratigraphie et préhistoire dans les 160 derniers millénaires en Limagne d'Auvergne (Massif Central, France), Bulletin de la Société Prónistorique Française, t. $91, n^{\circ} 2$, p. 149-157.

SACCHI D. (1986) - Le Paloolithlque superieur du Languedoc occidental et du Roussillon, XX|' supplément a Gallia. Préhistoire. Paris, C.N.R.S.

Surmely F. (1993) - Le mammouth, géant de la préhistoire. Paris, éditions Solar, $147 \mathrm{p}$.

SuAmely F. (1995 a) - Cors, un site magdalénien de la moyenne montagne cantalienne. In : Vivre on moyenne montagne, actes du colloque de Cler-
mont-Ferrand. Paris, editions du C.T.H.S., p. $65-87$.

Surmel. F. (1995 b) - Le peuplement des Limagnes et de leurs marges au tardiglaciaire. Livret-guide de l'AFEQ. Clermont-Ferrand, p. 11-15, dact.

TABofin Y. (1970) - Quelques objets de parure. Etude technologique : le percement des incisives de bovinés et des canines de renards. In : Deuxième colloque international sur l'industrie de l'os dans la prénistoire. Senanque. C.N.R.S., p. $303-310$

TORTI Ch. (1980) - Recherches sur l'implantation humaine en Limagne au pa. teolithique moven et supenieur. These de l'université de Bordeaux I, 2 vol, dact.

VERNET G. (1992) - Message du volcanisme régional dans les formations quaternaire de Limagne occidentale (Massif Central trançais). Minéraux denses et retombees. Thèse de l'Université de Bordeaux I, $n^{\circ} 724,335 \mathrm{p}$.

Frédéric SURMELY Service Rogional de l'Archéologie d'Auvergne 4, rue Pascal, 63000 Clemnont-Ferrand U.R.A. 1562 et U.M.R. 9933

Laure FONTANA U.R.A. 1415 Laboratoire d'Anatomie Comparé 55, rue Buffon, 75005 Paris

Yves BOURDELLE 14, rue des Beaumes 63100 Clermont-Ferrand

René LIABEUF Service Régional de I'Archeologie d'Auvergne 